\title{
Basins of attraction of the bistable region of time-delayed cutting dynamics
}

\author{
Yao Yan* \\ School of Aeronautics and Astronautics, University of Electronic Science and Technology of China, Chengdu 611731, China \\ Jian $\mathrm{Xu}^{\dagger}$ \\ School of Aerospace Engineering and Applied Mechanics, Tongji University, Shanghai 200092, China

\begin{abstract}
Marian Wiercigroch ${ }^{\ddagger}$
Centre for Applied Dynamics Research, School of Engineering, King's College, University of Aberdeen, Aberdeen, AB24 $3 U E$ Scotland, UK
\end{abstract}

(Received 24 May 2017; published 6 September 2017)

\begin{abstract}
This paper investigates the effects of bistability in a nonsmooth time-delayed dynamical system, which is often manifested in science and engineering. Previous studies on cutting dynamics have demonstrated persistent coexistence of chatter and chatter-free responses in a bistable region located in the linearly stable zone. As there is no widely accepted definition of basins of attraction for time-delayed systems, bistable regions are coined as unsafe zones (UZs). Hence, we have attempted to define the basins of attraction and stability basins for a typical delayed system to get insight into the bistability in systems with time delays. Special attention was paid to the influences of delayed initial conditions, starting points, and states at time zero on the long-term dynamics of time-delayed systems. By using this concept, it has been confirmed that the chatter is prone to occur when the waviness frequency in the workpiece surface coincides with the effective natural frequency of the cutting process. Further investigations unveil a thin "boundary layer" inside the UZ in the immediate vicinity of the stability boundary, in which we observe an extremely fast growth of the chatter basin stability. The results reveal that the system is more stable when the initial cutting depth is smaller. The physics of the tool deflection at the instant of the tool-workpiece engagement is used to evaluate the cutting safety, and the safe level could be zero when the geometry of tool engagement is unfavorable. Finally, the basins of attraction are used to quench the chatter by a single strike, where the resultant "islands" offer an opportunity to suppress the chatter even when the cutting is very close to the stability boundary.
\end{abstract}

DOI: 10.1103/PhysRevE.96.032205

\section{INTRODUCTION}

Bistability or multistability has been universally found in almost all areas of science and engineering, including neuronal systems [1], optics [2], electronics [3], epidemics [4], and mechanical systems [5]. This discovery has been an exciting development for the dynamical systems research community as it offers an opportunity to control the system dynamics without the need to vary the system parameters [6]. However, the coexistence of various attractors for a given set of parameters also poses a challenge to drive the system dynamics to a desired attractor. This is because any unexpected perturbation may move the system to another undesired state [7]. Therefore, to estimate the global robustness of each attractor, the current approach is to compute basins of attraction to predict the long-term behavior [8]. Correspondingly, the likelihood of the system reaching any state can be estimated by its basin stability [9].

When bistability emerges in time-delayed systems, the conventional basins of attraction approach is not applicable, as a time-delayed system has infinite dimensions [10]. To address this problem, various definitions of basins of attraction for delayed systems have been proposed including those present in Refs. [11-15]. For example, Wang et al. [11] introduced

\footnotetext{
*y.yan@uestc.edu.cn

†xujian@tongji.edu.cn

${ }^{\ddagger}$ m.wiercigroch@abdn.ac.uk
}

polynomials to approximate the functional initial conditions and used the coefficients of polynomials to construct the basins of attraction of a Duffing oscillator with delayed feedbacks. In the discussion of global dynamics of a van der Pol equation with time-delayed feedback control, Höhne et al. [12] switched off the control force at the beginning to avoid a dilemma of selecting a function of time to choose the initial conditions. Thus, the basins of attraction for such a system only depend on the very initial instant $(t=0)$. To control the erosion of safe basins in a damped Mathieu-Duffing oscillator with a single-well potential, Shang and $\mathrm{Xu}$ [13] successively introduced delayed position, velocity, and state feedbacks, with the delayed initial states (the states before time $t=0$ ) fixed at zero. In a similar manner, by defining the initial conditions as constants instead of functions of time, Ji [14] has observed the basins of attraction of small- and large-amplitude oscillations in a nonlinear system with time-delayed feedback. For a delayed Hopfield neuronal model, its function of initial values was expanded along with various orthogonal bases by Leng et al. [15], who confirmed that the selection of those bases is not important as long as the number of the basis functions is large enough.

Up to now, most discussions of the bistability in timedelayed systems have been rather artificial as delays were coming from control signals. For a different control strategy, delayed initial conditions have been hypothetically chosen as zero, constant, polynomial, or orthogonal. However, it should be noted here that many physical problems have inherent time delays such as cutting dynamics [16], traffic dynamics [17], 
neural dynamics [18], internet systems [19], laser generators [20]. In such systems, one cannot select a priori initial signal for the delayed terms. When Levnajić studied the basin stability of phase-repulsive networks of oscillators with coupling time delays, he had to assume no delay for the initial conditions [21,22]. Therefore, to make progress in the analysis of nonlinear dynamics with time delay and bistability, this paper focused on a general problem of tracking and controlling of bistability of a simple dynamical system for which time delays and bistability are inherent.

In metal cutting dynamics, there are nonlinear dynamical systems that have inherent time delays presenting bistability. They originate from cutting processes, including turning [16], milling [23], drilling [24], and grinding [25]. Moreover, it has been found that the phenomenon of bistability persists in every cutting processes, which can compromise cutting accuracy and safety in the bistable regions regarded as unsafe zones (UZs) $[23,26,27]$. Although the location and size of the UZs have been successfully estimated [28], time delays always hamper the analysis of basin stability, so detailed investigations of long-term dynamics inside the UZs are often problematic.

To systematically deepen our understanding of the bistability in time-delayed systems in this paper, we study the basin stability of the UZs in a typical regenerative cutting process structured in the following sections. First, Sec. II briefly reviews the main investigations of the bistability and the UZs in the cutting processes [27]. Then, Sec. III employs the orthogonal basis to represent the initial waviness of the outmost layer in the workpiece surface to study its profile affecting the probability of chatter occurrence. Next, the cutting geometry is found to be a key parameter influencing the basin stability of the chatter, which is studied in Sec. IV. In Sec. V, the effect of tool deformation on the basin stability is analysed, and the cutting safety is estimated by three different measures. Next, by switching between different attractors, a control law for quenching the chatter in the UZs by a single strike is proposed in Sec. VI. Finally, Sec. VII draws some conclusions.

\section{UNSAFE CUTTING DUE TO BISTABILITY}

Many physical and engineering systems are governed by nonlinear delayed differential equations (DDEs), and cutting process is a typical and widely investigated example [26]. Due to nonlinearity in cutting forces, a cutting process is often accompanied by coexistence of stable and unstable responses in bistable regions (UZs). Coexistence of stable and unstable responses in UZs induced by a subcritical Hopf bifurcation has been found in various machining processes described by DDEs, but the risk associated with a cutting process operating in the UZs is still unknown. To address this issue, a new measure is required to estimate cutting safety or probability of chatter occurrence. A new concept of this measure will be presented in the following analysis based on a typical onedimensional regenerative turning process.

The simplest physical model of the regenerative metal cutting is depicted in Fig. 1, which dynamics is governed by

$$
\ddot{x}(T)+2 \xi \omega_{\mathrm{n}} \dot{x}(T)+\omega_{\mathrm{n}}^{2} x(T)=f(T),
$$

where $\omega_{\mathrm{n}}=\sqrt{k / m}, \xi=c /\left(2 m \omega_{\mathrm{n}}\right), m, c$, and $k$ are natural frequency, damping ratio, mass, damping coefficient, and

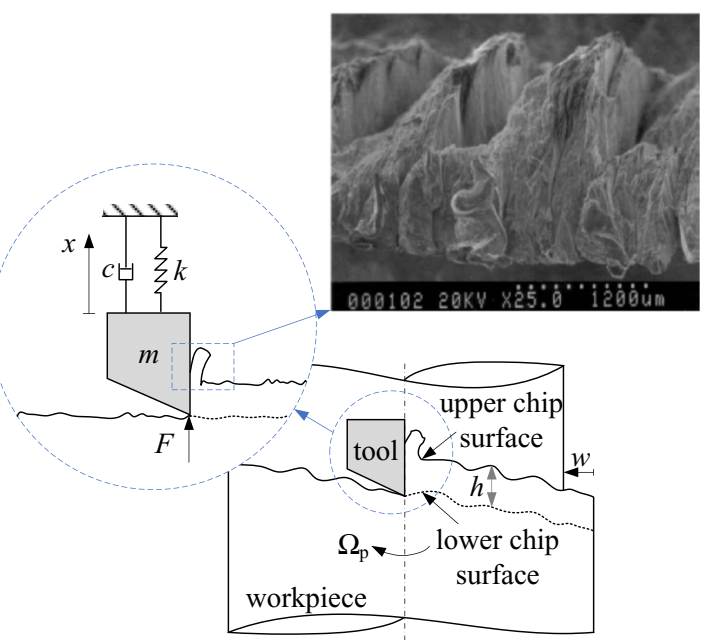

FIG. 1. Physical model of the cutting process, with an SEM (scanning electron microscopy) image of the chips formation [29], which generates cutting forces.

stiffness of the tool, respectively. The tool is excited by the cutting force, $f(T)$, given by [27]

$$
f(T)=\frac{w}{m}\left[\rho_{1} h(T)+\rho_{2} h(T)^{2}+\rho_{3} h(T)^{3}\right],
$$

where $w$ and $h(T)$ are chip width and instantaneous cutting depth, and $\rho_{1}, \rho_{2}$, and $\rho_{3}$ are coefficients determined from experiments [30]. $h(T)$ is a sum of the nominal cutting depth $h_{0}$ provided by the tool feed per revolution and the current tool displacement $x(T)$. In addition, as the upper chip surface (see the top right panel of Fig. 1) was generated by the previous tool pass, which should be accounted for in the cutting depth as well. That is $h(T)=h_{0}+x\left(T-T_{\mathrm{p}}\right)-x(T)$, where $T_{\mathrm{p}}=$ $2 \pi / \Omega_{\mathrm{p}}[\mathrm{s}]$ is the period of workpiece rotation. Simply put, the tool motion is governed by a typical nonlinear DDE.

In a more general sense, it is worth noting that Eq. (1) is the simplest model governing the cutting dynamics, which is simply a linear oscillator subjected to delayed force with square and cubic nonlinearities. By studying this fundamental system, one can get insight not only into the unsafe cutting but also into other dynamics involving both bistability and time delays.

Given Eq. (2), Eq. (1) is nondimensionalized by using the following dimensionless variables and parameters:

$$
\begin{aligned}
t & =\omega_{n} T, \quad d=\frac{h}{h_{0}}, \quad \tau=\omega_{n} T_{p}, \quad \Omega=\frac{\Omega_{p}}{\omega_{n}}, \\
y_{1} & =\frac{x}{h_{0}}-\frac{w}{m \omega_{n}^{2}}\left(\rho_{1}+h_{0} \rho_{2}+h_{0}^{2} \rho_{3}\right), \quad y_{2}=\frac{d y_{1}}{d t} \\
\eta_{1} & =\frac{w}{m \omega_{n}^{2}}\left(\rho_{1}+2 h_{0} \rho_{2}+3 h_{0}^{2} \rho_{3}\right), \\
\eta_{2} & =\frac{w}{m \omega_{n}^{2}}\left(h_{0} \rho_{2}+3 h_{0}^{2} \rho_{3}\right), \quad \eta_{3}=\frac{w}{m \omega_{n}^{2}} h_{0}^{2} \rho_{3},
\end{aligned}
$$

which yields

$$
\mathbf{y}^{\prime}(t)=\mathbf{A y}(t)+\mathbf{D y}(t-\tau)+\mathbf{N}
$$




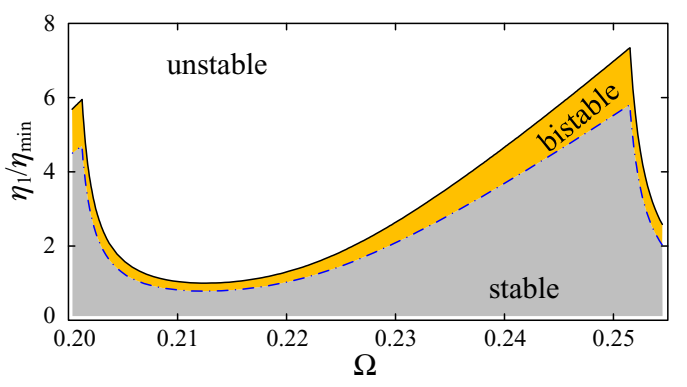

FIG. 2. Stability boundaries (solid black) and boundaries for the UZ obtained by DDEBIFTOOL (dot-dashed blue).

where the prime denotes the derivatives with respect to the dimensionless time $t$, and

$$
\begin{aligned}
\mathbf{y}(t) & =\left(\begin{array}{l}
y_{1} \\
y_{2}
\end{array}\right), \quad \mathbf{A}=\left(\begin{array}{cc}
0 & 1 \\
-1-\eta_{1} & -2 \xi
\end{array}\right), \quad \mathbf{D}=\left(\begin{array}{cc}
0 & 0 \\
\eta_{1} & 0
\end{array}\right), \\
\mathbf{N} & =\left(\begin{array}{l}
0 \\
\eta_{2}\left[y_{1}(t-\tau)-y_{1}(t)\right]^{2}+\eta_{3}\left[y_{1}(t-\tau)-y_{1}(t)\right]^{3}
\end{array}\right) .
\end{aligned}
$$

Next, the eigenvalue analysis of Eq. (4) results in the characteristic equation, $\operatorname{det}\left(\lambda \mathbf{I}-\mathbf{A}-\mathbf{D} e^{-\lambda \tau}\right)=0$, where $\operatorname{det}(\cdot)$ is the determinant of $\cdot$ and $\lambda$ is the eigenvalue. A stable stationary cutting process requires the real parts of all the eigenvalues to be negative $[\operatorname{Re}(\lambda)<0]$, while any positive real part would generate unstable cutting. For the points on the stability boundaries, purely imaginary complex conjugate roots $\lambda= \pm i \omega$ can be found, yielding parametric functions of $\omega[27,31]$, such as

$$
\begin{aligned}
\eta_{1 c}(\omega) & =\frac{\left(\omega^{2}-1\right)^{2}+4 \xi^{2} \omega^{2}}{2\left(\omega^{2}-1\right)}, \\
\tau_{c}(\omega) & =\frac{2}{\omega}\left[i \pi-\arctan \left(\frac{\omega^{2}-1}{2 \xi \omega}\right)\right],
\end{aligned}
$$

where $i=1,2,3, \cdots$ and $\omega \in(1,+\infty)$. Here, $\eta_{1 c}$ and $\tau_{c}$ denote the critical values of $\eta_{1}$ and $\tau$ on the stability boundaries. In addition, the minimum value of $\eta_{1 c}$ on each boundary is $\eta_{\min }=\min \left[\eta_{1 c}(\omega)\right]=2 \xi(1+\xi)$ [27].

The same as Refs. [27,30], the boundaries given by Eq. (5) for $\xi=0.01, \rho_{1}=6109.6, \rho_{2}=-54141.6$, and $\rho_{3}=$ 203769 are marked as the solid black curves in Fig. 2, where $\Omega=2 \pi / \tau$ is the dimensionless angular velocity of the workpiece. The white region over the curves in Fig. 2 is unstable while the grey and yellow areas are linearly stable [32]. A further nonlinear dynamic analysis reveals bistability in the yellow region (UZs), which has been computed with DDEBIFTOOL by following an unstable limit cycle born on the boundary until the loss of tool-workpiece contact occurs [26,27].

To predict long-term dynamics in bistable regions an intuitive method is to compute basins of attraction, which is straightforward for ordinary differential equations (ODEs). However, there is no widely accepted definition of basins of attraction for DDEs, since the corresponding initial conditions are defined in a functional space [10]. As a result, the initial conditions for discussion of the long-term cutting dynamics in the UZs should be defined beforehand according to the physical meanings of the delayed terms [33].

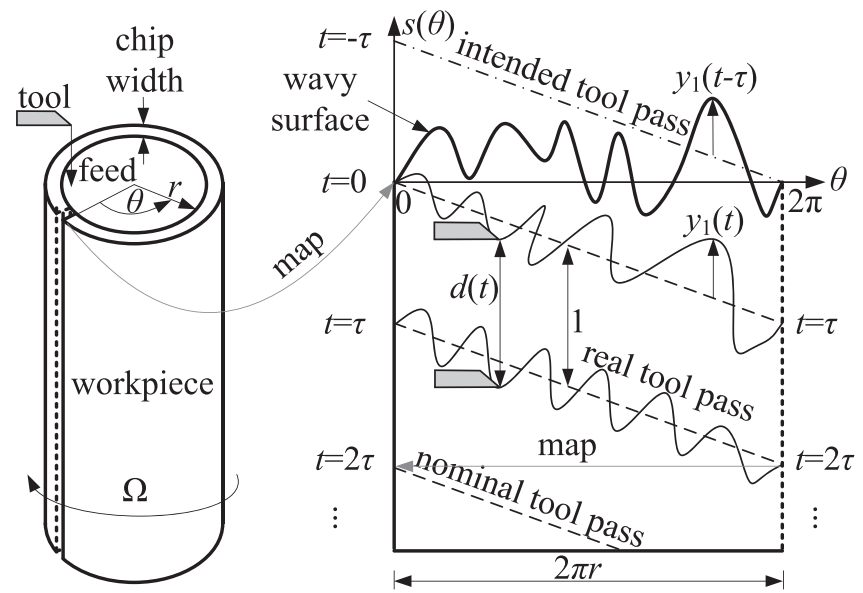

FIG. 3. Outmost layer of the workpiece is mapped onto the $\theta-s(\theta)$ plane with a thick solid curve for the surface profile and the coordinate origin for the beginning of the tool-workpiece engagement. Thin dashed lines, thin solid curves, and a dot-dashed line are for nominal, real, and intended tool passes, respectively. Every time $\theta$ reaches $2 \pi$, the tool position is mapped back to $\theta=0$.

\section{SURFACE WAVINESS}

The delayed initial condition, $y_{1}(t)(t \in[-\tau, 0])$, represents system state before time zero. For systems with time-delayed controllers, the state is determined by control signals and can be artificially adjusted as described in Refs. [13,14]. For artificial neuron networks, the state is given by associative memory, which is gained by training the networks to recall when similar events occur [15]. For metal cutting, the state is related to the initial surface waviness, which can be deemed as the workpiece's "memory" of previous processing, i.e., the surface records the delayed initial conditions of the cutting dynamics.

For illustration, Fig. 3 shows successive removals of the outmost layers of the workpiece. The cutting tool penetrates into the workpiece at the left edge of the layer (thick solid line), then moves rightwards until it reaches the right edge (thick dashed line), where the tool is mapped back to the left edge. On the layer, nominal and real tool passes are plotted in thin dashed and solid curves, which have the difference $y_{1}(t)$. The workpiece has an original wavy surface marked as a thick solid curve. If the initial wavy surface is regarded as the real tool pass during $t \in[-\tau, 0]$, an intended tool pass (dot dashed) is added as an auxiliary line for the initial condition, $y_{1}(t)(t \in[-\tau, 0])$.

By using the polar coordinate, $\theta \in[0,2 \pi]$, the workpiece surface can be described by a periodic function, $s(\theta)$, which has $s(\theta+2 \pi)=s(\theta)$. Meanwhile, the intended pass is a line segment given by $p(\theta)=1-\frac{\theta}{2 \pi}(\theta \in[0,2 \pi])$. Combining them yields the initial condition $y_{1}(\theta)=s(\theta)-p(\theta)$ $(\theta \in[0,2 \pi])$. Given the workpiece angular rotation and the tool feed, the polar coordinate can be represented as $\theta=2 \pi+\frac{t}{\tau} 2 \pi=2 \pi+\Omega t(t \in[-\tau, 0])$, which gives

$$
y_{1}(t)=s(2 \pi+\Omega t)+\frac{\Omega t}{2 \pi}=s(\Omega t)+\frac{\Omega t}{2 \pi}, \quad t \in[-\tau, 0] .
$$


(a)

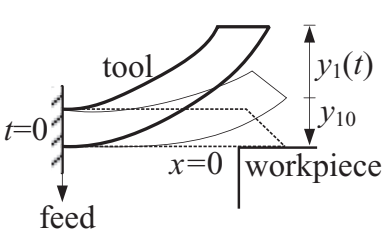

(b)

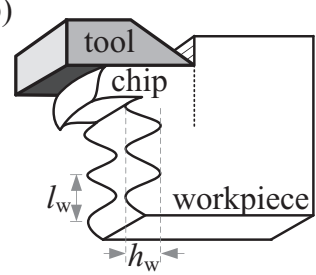

FIG. 4. (a) Tool deflection as it penetrates into the workpiece; the tool equilibrium is moved from $x=0\left(y_{1}=-y_{10}\right)$ to $y_{1}=0$. (b) Initial surface profile.

Moreover, the initial condition includes the change of tool deflection during the penetration, which moves the tool equilibrium. As can be seen from Fig. 4(a), the tool does not bend $(x(0)=0)$ before it engages with the workpiece. From Eq. (3) one has $y_{1}=-\frac{w}{m \omega_{n}^{2}}\left(\rho_{1}+h_{0} \rho_{2}+h_{0}^{2} \rho_{3}\right)=-\left(\eta_{1}-\eta_{2}+\eta_{3}\right)$ when $x=0$. By denoting $y_{10}=y_{1}(0)=-\left(\eta_{1}-\eta_{2}+\eta_{3}\right)$, the initial condition becomes

$$
y_{1}(t)=s(\Omega t)+\frac{\Omega t}{2 \pi}+y_{10}, \quad t \in[-\tau, 0] .
$$

In addition, it is required that $s(0)=0$ at the instant of the toolworkpiece engagement $(t=0)$ because $y_{10}=y_{1}(0)=s(0)+$ $y_{10}$.

To sum up, $s(t)(t \in[-\tau, 0])$ is periodic as $s(-\tau)=s(0)=$ 0 , which can be expressed in an orthogonal trigonometric basis as [15]

$$
s(t)=\sum_{N=1}^{\infty} a_{N} \sin (N \Omega t),
$$

where $N$ is the frequency multiplier and $a_{N}$ the corresponding amplitude of each harmonic function.

Given the surface profile of the workpiece shown in Fig. 4(b), $a_{N}$ and $N$ are related to the waviness height $h_{w}$ and wavelength $l_{w}$ by $a_{N}=\frac{h_{w}}{2}$ and $N=\frac{2 \pi r_{w}}{l_{w}}$, where $r_{w}$ is the workpiece radius. In combination with the workpiece rotation, $a_{N}$ and $N \Omega$ can be deemed as amplitude and frequency of the waviness. Therefore, to understand the influence of the surface profile on the long-term cutting dynamics in the UZs, the two parameters, $N$ and $a_{N}$, are critical.

In a similar study, Leng et al. [15] have confirmed that the fluctuations of basin stability tend to be stable when $N$ is sufficient large. In this study, we also observe the same convergence when $N>10$. Correspondingly, the first basin of attraction is defined on $N-a_{N}$ plane, which includes the lowest 12 harmonics $(N=1,2, \ldots, 12)$ and the waviness amplitude for $a_{N} \in[0,4]$. To illustrate the point, the case for $\Omega=0.23$ and $\eta_{c 1} / \eta_{\min }=2.62$ is shown in Fig. 5(b), where the black regions mark the initial conditions leading to the regenerative chatter and the white the stable cases.

As reported by Molnár et al. [26], numerical simulation of Eq. (4) may yield chatter vibration with amplitude tending to infinity, since Eq. (4) dose not includes nonlinearities such as loss-of-contact, impact, etc. Therefore, the black regions in Fig. 5 mark the unstable cutting with either bounded or unbounded amplitude.

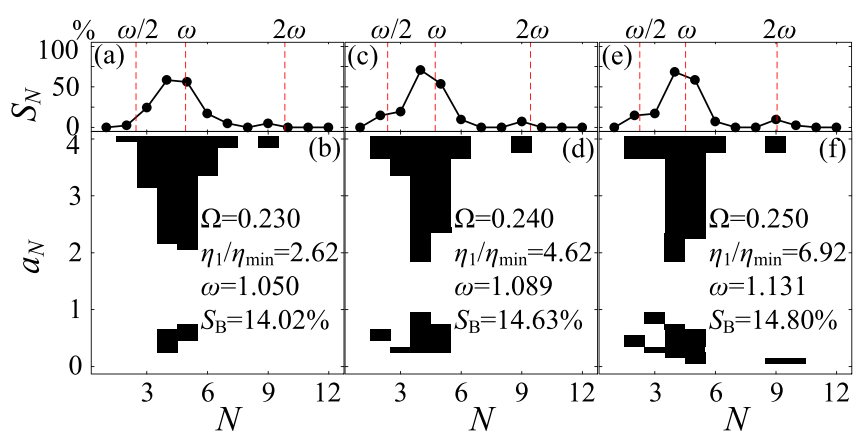

FIG. 5. Basins of attraction and basin stability for (a, b) $\Omega=$ 0.230 and $\eta_{1} / \eta_{\min }=2.620$, (c, d) $\Omega=0.240$ and $\eta_{1} / \eta_{\min }=4.620$, and (e, f) $\Omega=0.250$ and $\eta_{1} / \eta_{\min }=6.920$, where the the peaks of $S_{N}$ always coincides with the nature frequency $\omega$.

Corresponding to the basins of attraction, the overall basin stability of the chatter is defined as

$$
S_{B}=\frac{A_{u}}{A_{s}+A_{u}} \times 100 \%,
$$

where $A_{u}$ and $A_{s}$ represent the areas of the black (unstable) and white (stable) regions respectively. Specifically, the case in Fig. 5(b) has basin stability of $S_{B}=14.02 \%$, which means this cutting has the probability of $14.02 \%$ to experience chatter.

In addition, the basins stability for each harmonics, $S_{N}$ $(N=1,2, \cdots, 12)$, are calculated individually, with the corresponding distribution presented in Fig. 5(a). Substituting $\Omega=0.23$ into Eq. (5) yields the natural frequency of this cutting, $\omega=1.050$. The curve in Fig. 5(a) has two local peaks, one between $N=4$ and $5[N \Omega \in(0.92,1.15)]$ and another one near $N=9(N \Omega=2.07)$, which match the primary and secondary harmonics of the natural frequency, $\omega=1.05$ and $2 \omega=2.10$, respectively. The major peak near the primary frequency is very intuitive and means the oscillation is prone to occur when the initial states is near the chatter trajectory. By contrast, the minor peak near the superharmonics is unexpected and offers a new option for driving the system dynamics to the oscillating attractor. It should be remarked here that this phenomenon only occurs for DDEs, because the initial conditions for ODEs do not depend on frequency.

A similar phenomenon has been observed in Figs. 5(d)$5(\mathrm{f})$, where $\omega$ is varied with respect to the change of $\Omega$ along the stability boundary, but the peaks of $S_{N}$ always coincide with the corresponding natural frequency. In Fig. 5, no matter what frequency is chosen, e.g., $\Omega=0.230,0.240$, or 0.250 , as long as $\eta_{1} / \eta_{\min }$ is selected properly to ensure $S_{B} \approx 14 \%$, the patterns of the basin of attraction in $N-a_{N}$ plane are very similar and have the largest black region when $a_{N}$ is large and $N \Omega$ is near $\omega$, as well as some small black regions below or on the right side of it. To sum up, the regenerative chatter has a higher probability to occur when the workpiece has a waviness frequency $N \Omega$ coinciding with the natural frequency of the cutting $\omega$ and (or) its superharmonics.

When $\Omega$ is fixed, the value of $\eta_{1} / \eta_{\min }$ determines the basin stability $S_{B}$. Specifically, a large enough $\eta_{1} / \eta_{\min }$ ensures the chatter occurrence in the unstable zone $\left(S_{B}=100 \%\right)$, while a small enough $\eta_{1} / \eta_{\text {min }}$ guarantees the cutting stability $\left(S_{B}=0 \%\right)$. The UZ as a transitional region should witness a 

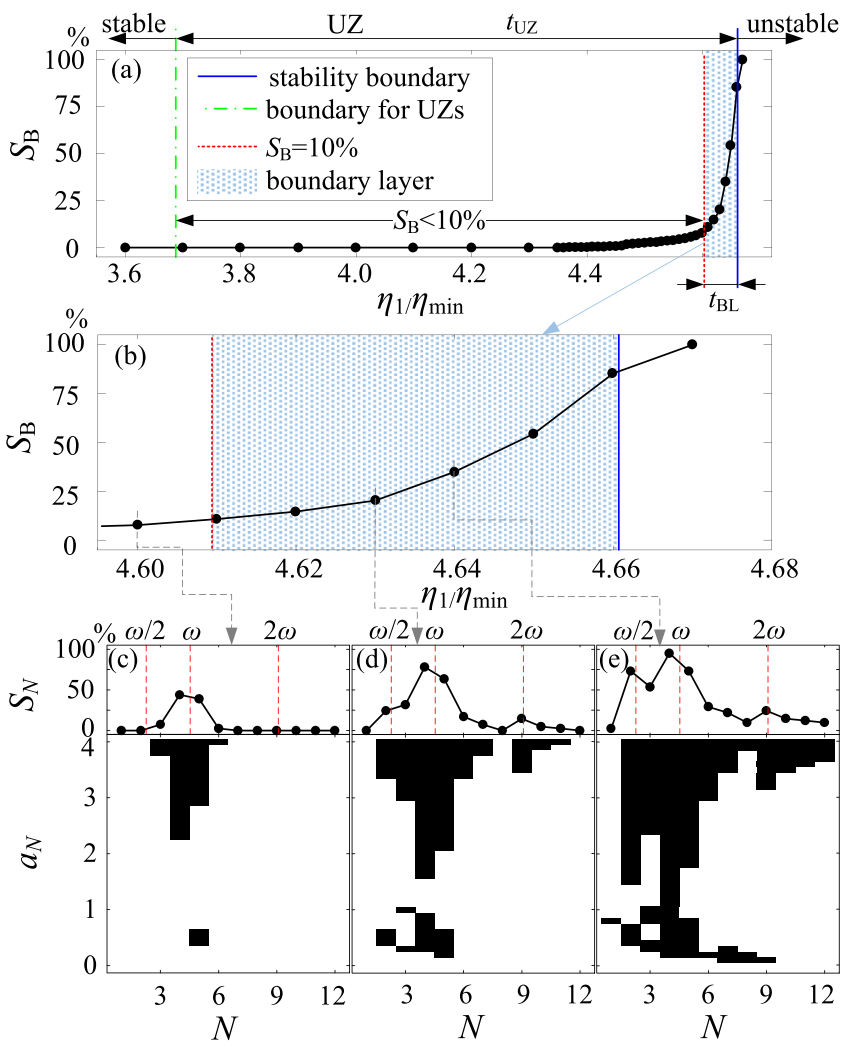

FIG. 6. (a) Overall basin stability $S_{B}$ as a function of $\eta / \eta_{\min }$ for $\Omega=0.24$, with the regions for a fast growth of $S_{B}$ been blown up in panel (b). (c-e) Basins of attraction and basin stability distributions for $\eta_{1} / \eta_{\min }=4.60,4.63$, and 4.64 .

continuous increase of $S_{B}$ from $0 \%$ to $100 \%$. The transition for $\Omega=0.240$ and $\eta_{1} / \eta_{\min } \in[3.6,4.7]$ is illustrated in Fig. 6, where Fig. 6(a) shows the growth of $S_{B}$ with respect to the increase of $\eta_{1} / \eta_{\min }$, which is very slow for $\eta_{1} / \eta_{\min }<4.6$ but extremely fast thereafter. The region for the fast increase is blown up in Fig. 6(b), with the corresponding basins of attraction for $\eta_{1} / \eta_{\min }=4.60,4.63$, and 4.64 added. Figure 6(c) displays a low probability of chatter, with a very small region around $N \Omega \approx \omega$. Then, increase of $\eta_{1} / \eta_{\text {min }}$ to 4.63 enhances the probability of chatter occurrence and introduces a new small peak at $N \Omega \approx 2 \omega$. Next, $\eta_{1} / \eta_{\text {min }}=4.64$ further enlarges the black region and introduces the third peak at $N \Omega \approx \omega / 2$, which coincides with the subharmonic of the natural frequency. Thereafter, the black region spreads to gradually occupy the whole $N-a_{N}$ plane when $\eta_{1} / \eta_{\min }$ keeps increasing to reach the end of the transitional region (UZ): $\eta_{c 1} / \eta_{\min }$.

The UZ in Fig. 6(a) shows steady growth of $S_{B}$ when $\eta_{1} / \eta_{\text {min }}$ is far away from $\eta_{c 1} / \eta_{\text {min }}$ but burgeoning basin stability when $\eta_{1} / \eta_{\min }$ approaches the boundary, which is similar to the phenomenon called "boundary layer." To illustrate this, a dashed red line is added to marked the position for $S_{B}=10 \%$, and the region between the dashed red and solid blue (where $\eta_{1}=\eta_{c 1}$ ) lines is defined as the "boundary layer." As can be seen, the area between the dot-dashed blue (boundary of the UZ) and dashed red lines occupy most of the UZ (roughly 94\%), but it only has no more than $10 \%$ probability of the chatter occurrence. When the relative thickness of the

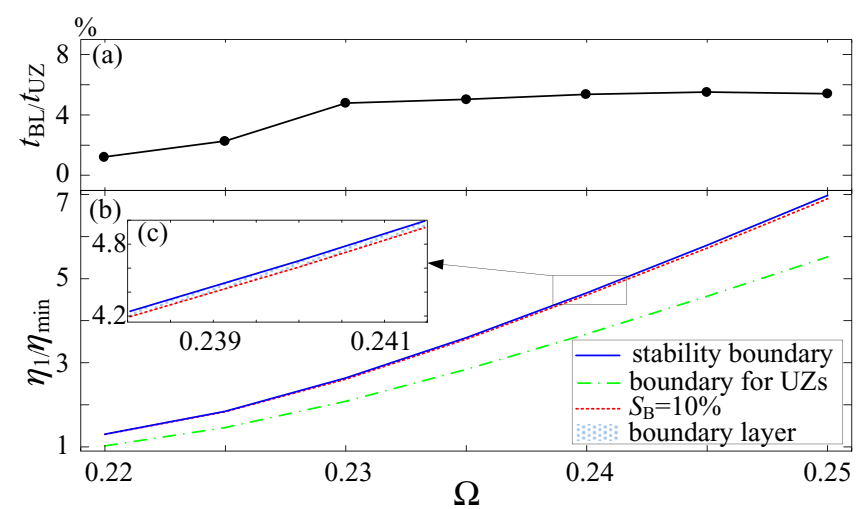

FIG. 7. (a) Relative thickness of the "boundary layer" (b) and its location in the $\Omega-\eta_{1} / \eta_{\min }$ plane.

"boundary layer" is defined as $t_{\mathrm{BL}} / t_{\mathrm{UZ}}$, where $t_{\mathrm{BL}}$ and $t_{\mathrm{UZ}}$ are the thickness of the "boundary layer" and the UZ, Fig. 6(a) only has $t_{\mathrm{BL}} / t_{\mathrm{UZ}}<6 \%$. Similar results are observed all along the stability boundary for $\Omega \in[0.22,0.25]$, which shows no more than $6 \%$ relative thickness for the thin "boundary layer" in Fig. 7.

The peaks in the distribution of $S_{N}$ show how to drive the dynamics a time-delayed system to the oscillating orbit, which chooses the initial conditions having the frequency coinciding with the frequency of the orbit and its super- and subharmonics. The phenomenon of the "boundary layer" could be beneficial because it lowers the probability of chatter occurrence when the cutting process has been designed in the UZs, which eases our worry about the accuracy of linear stability analysis.

\section{GEOMETRY OF TOOL ENGAGEMENT}

For any time-delayed dynamics, the "memory" is fixed, but the starting point is undetermined. For example, when a time-delayed network is stimulated at time zero, its future dynamics is determined by the states in the time span before the stimulation (the delayed initial conditions). As a result, the moment of stimulation becomes very critical as it determines which part of the "memory" is read. Similarly, when the tool cuts into different points in the workpiece surface, different delayed initial conditions are yielded by the same waviness. This is illustrated in Fig. 8, where the original wavy workpiece surface is represented by $s(\theta)$. Given the workpiece rotation and the tool feed, the cutting could start (for $t=0$ ) at any point on the circumference of the

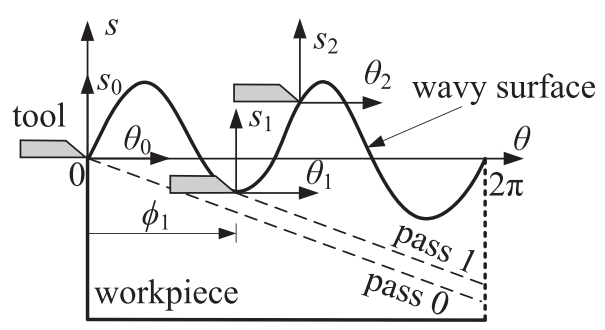

FIG. 8. Different points of penetration yield different tool passes and coordinates of $\theta-s$. 

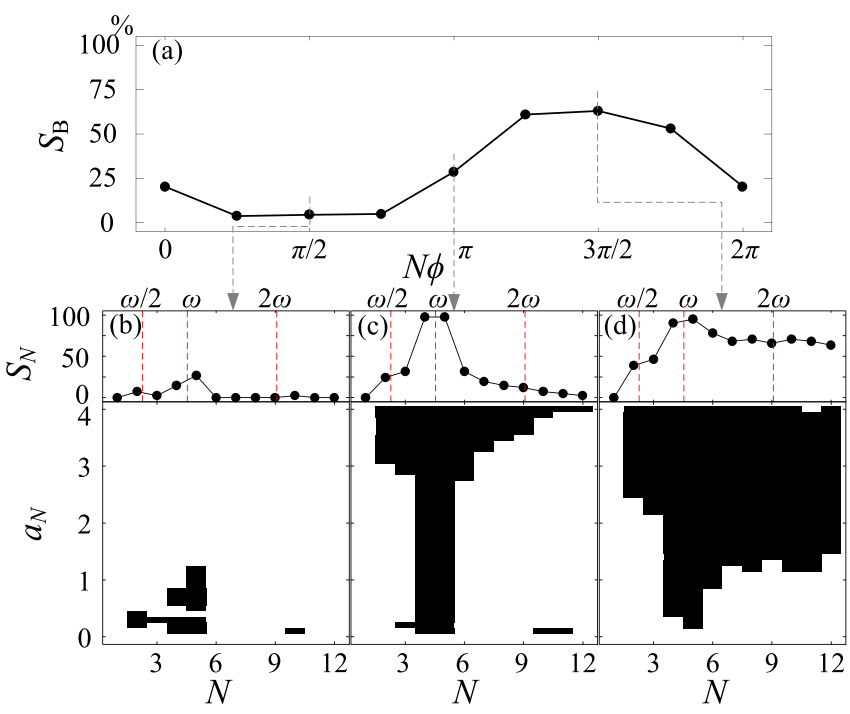

FIG. 9. (a) Influence of geometry of tool engagement on the basin stability for $\Omega=0.240$ and $\eta_{1} / \eta_{\min }=4.630$, with basin stability distributions and basins of attraction for (b) $\pi / 2$, (c) $\pi$, and (d) $3 \pi / 2$ added for demonstration.

workpiece $(\theta \in[0,2 \pi])$. For example, the engagement at $\theta=0$ yields the coordinates, $\theta_{0}=\theta$ and $s_{0}=s$, and pass 0 for the tool movement. By contrast, if the penetration happens at $\theta=\phi_{1}, \theta_{1}-s_{1}$, and pass 1 should be used to describe the wavy surface and the cutting path. Apparently, $\theta_{1}=\theta-\phi_{1}$ and $s_{1}\left(\theta_{1}\right)=s(\theta)-s\left(\phi_{1}\right)=s\left(\theta_{1}+\phi_{1}\right)-s\left(\phi_{1}\right)$. The same as $s_{0}\left(\theta_{0}\right), s_{1}\left(\theta_{1}\right)$ is also periodic since $s_{1}\left(\theta_{1}\right)=s_{1}\left(\theta_{1}+2 \pi\right)$ and has $s_{1}(0)=s_{1}(2 \pi)=0$. Without loss of generality, we drop the subscripts of $s_{1}, \theta_{1}$, and $\phi_{1}$, and let $N \phi \in[0,2 \pi)$, and thus change the basis for the initial condition, Eq. (8), into

$$
s(t)=\sum_{N=1}^{\infty} a_{N}[\sin (N \Omega t+N \phi)-\sin (N \phi)] .
$$

Given Eq. (10), a typical result for $\Omega=0.240$ and $\eta_{1} / \eta_{\min }=4.630$ is depicted in Fig. 9, in which Fig. 9(a) shows that $N \phi=0$ and $2 \pi$ yield the same result, since Eq. (10) is a periodic function of $N \phi$. With respect to the increase of $N \phi$ in the domain of $[0,2 \pi), S_{B}$ first undergoes a descent until it reaches its lowest point at $N \phi=\pi / 2$, and then starts to grow for its maximum at $N \phi=3 \pi / 2$, which commences another decrease thereafter. In addition, the basins of attraction and distribution of $S_{N}$ are plotted for $N \phi=0, \pi / 2, \pi$, and $3 \pi / 2$ in Figs. 9(b)-9(d), which show a similar pattern to those in Figs. 5 and 6 , confirming the previous statement that the probability of chatter occurrence $S_{N}$ reaches its maximum when $N \Omega$ is near $\omega$. Unlike other cases, Fig. 9(b) shows accumulation of the black regions at bottom-left part of the $N-a_{N}$ plane, but the region with large $a_{N}$ has no chatter. This accumulation reminds us that a large waviness height in the workpiece surface is not a necessary condition to incur the chatter. Therefore, we should focus on the waviness frequency instead of the height for avoiding cutting chatter.

The result shown in Fig. 9(a) is further demonstrated in Fig. 10, in which $\Omega$ is fixed at 0.240 and $\eta_{1} / \eta_{\min }$ varies between 4.32 and 4.67. The contour plot in Fig. 10(b) displays the basin

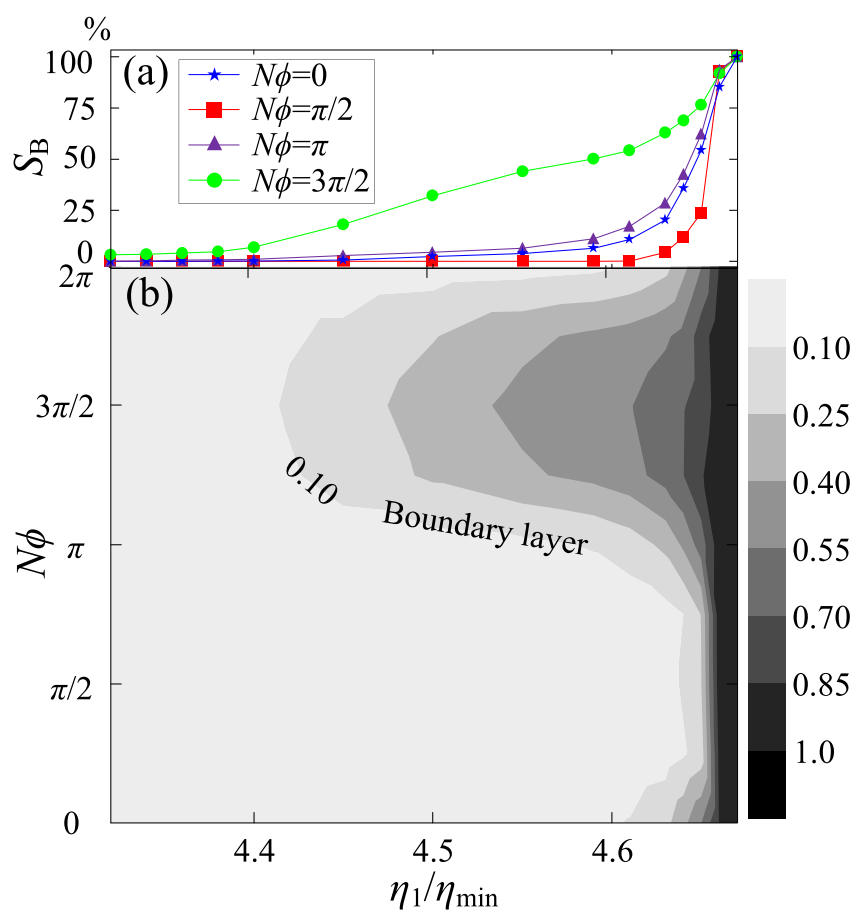

FIG. 10. (a) Basin stability $S_{B}$ is plotted as functions of $\eta_{1} / \eta_{\min }$ for $N \phi=0, \pi / 2, \pi$, and $3 \pi / 2$, respectively. (b) Contour plot of $S_{B}$ as a function of $\eta_{1} / \eta_{\min }$ and $N \phi$.

stability as a function of $N \phi$ and $\eta_{1} / \eta_{\min }$, where the darkest and the lightest color represent the highest and lowest possibilities of chatter occurrence. It is seen that the region around $N \phi=$ $3 \pi / 2$ is much darker than any other places, while the lightest region has the largest width when $N \phi$ approaches $\pi / 2$, which verifies the result in Fig. 9(a). In addition, the selected values of $S_{B}$ for $N \phi=0, \pi / 2, \pi$, and $3 \pi / 2$ are displayed in Fig. 10(a) as functions of $\eta_{1} / \eta_{\min }$, where the green line for $N \phi=3 \pi / 2$ is much higher than the other cases, so that the corresponding "boundary layer" is enlarged, which is not useful for practical applications.

In general, $N \phi=\pi / 2$ is preferred for avoiding cutting chatter, which is regarded as a "favorable geometry of tool engagement" compared with the "unfavorable" one for $N \phi=$ $3 \pi / 2$. The two cases are illustrated in Fig. 11, where the "favorable" case has a relatively small cutting depth when the tool starts to engage with the workpiece, and the "unfavorable" one induces a fast increase of the depth as soon as the tool

(a)

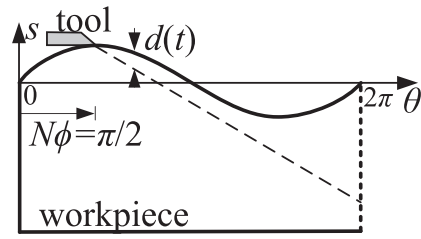

FIG. 11. Different geometry of tool engagement induce different cutting depth at the beginning of tool-workpiece interactions, where (a) favorable and (b) unfavorable geometry correspond to $N \phi=\pi / 2$ and $N \phi=3 \pi / 2$, respectively. 
penetrates into the workpiece. Since the source of cutting instability is the regenerative force, one can presume that a large cutting force at the beginning leads to a high probability of chatter occurrence.

The results illustrated in Figs. 9 and 10 reminds us that the starting point is important for driving a system to a desired trajectory. This is critical for switching between coexistence attractors, which will be used in Sec. VI to avoid chatter.

\section{TOOL BENDING}

The discussions in the previous sections have focused on the delayed initial conditions, $y_{1}(t)[t \in[-\tau, 0)]$. Moreover, the system state at time zero $\left[y_{1}(0), y_{2}(0)\right]$ also influences the final destination of the dynamic system. The conventional basins of attraction are all defined on the $\left[y_{1}(0), y_{2}(0)\right]$ plane and this is also used in our analysis of DDEs.

For the cutting system the given analysis so far assumes no tool bending at the instant of tool-workpiece engagement $[x(0)=0]$, which ignores the free tool vibration generated by the feeding motion. Given this effect, the initial state $\left[y_{1}(0), y_{2}(0)\right]$, which was assumed to be $\left(y_{10}, 0\right)$, becomes unknown. Correspondingly, the cutting safety with respect to the tool bending deflection can be evaluated by the basins of attraction defined on the $\left[y_{1}(0), y_{2}(0)\right]$ plane.

It is worth noting here that the third basin of attraction defined in this section is exactly the same as the conventional ones, so that many measures can be adopted [34]. We employ three different measures for the assessment, namely the global integrity measures (GIM), the integrity factor (IF), and the safe level (SL). GIM and IF are adopted from Refs. [35,36], which estimate the relative entire safe area and the relative area of the largest circle inscribed in the same safe area. SL determines the relative area of the largest inscribed circle centered at $\left[y_{1}(0), y_{2}(0)\right]=\left(y_{10}, 0\right)$, which is for the undeformed tool with no initial velocity. Therefore, SL actually evaluates the robustness of the ideal cutting geometry, which would not induce chatter as long as the unanticipated external perturbation is small enough (located in the circle).

The basins of attraction shown in Figs. 12(b)-12(g) were calculated, where the black and white regions are for the initial conditions leading to unstable and stable cutting, respectively. Given the basin stability of chatter defined as in Eq. (9), GIM = $100 \%-S_{B}$. Besides, the red and blue circles inscribed in the safe area (white) are for the IF and the SL, respectively. As a result, it can be seen in Fig. 12(a) that the largest measure is GIM, while SL yields the most conservative estimation of the cutting safety.

The influence of the cutting geometry on the three measures is displayed in Fig. 12(a), which shows a similar variation of GIM and IF with respect to $N \phi$, and this result corresponds to the variation of $S_{B}$ shown in Figs. 9 and 10. That largest GIM and IF are achieved when $S_{B}$ has the smallest value, while the smallest GIM and IF turn up when $N \phi$ is around $3 \pi / 2$, which means that $N \phi \approx \pi / 2$ is the safest point for the cutting geometry and $N \phi \approx 3 \pi / 2$ is the worst. By contrast, SL has slight differences compared with GIM and IF. Specifically, its maximum is achieved at $N \phi=\pi / 4$, which is in a slight advance of $\pi / 2$. Moreover, the minimum value of SL is 0 ,

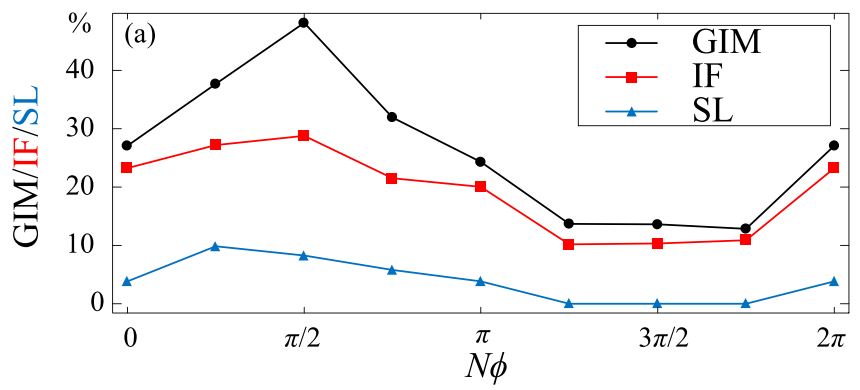

(b) $N \phi=0$



(e) $N \phi=3 \pi / 4$

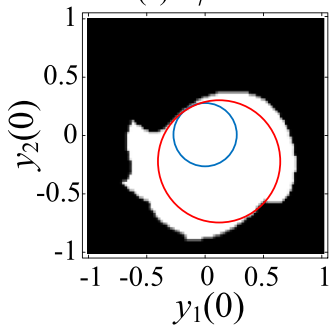

FIG. 12. (a) GIM, IF, and SL of the cutting vary as functions of the cutting geometry, with the basins of attraction for $N \phi=$ $0, \pi / 4, \pi / 2,3 \pi / 4, \pi, 3 \pi / 2$, and $5 \pi / 4$ plotted in panels $(\mathrm{b}-\mathrm{g})$. The rest of the parameters are selected as $\Omega=0.24, \eta_{1} / \eta_{\min }=4.60, N=$ 5 , and $a_{N}=1.0$.

which means the point $\left(y_{10}, 0\right)$ is in the black region and thus the ideal cutting is definitely unsafe.

In general, the influence of $N \phi$ on the basins of attraction is still periodic, so that $N \phi=0$ and $N \phi=2 \pi$ yield the same result. For $N \phi=0$, the safe basin (white region) is located on the left of the $y_{1}(0)-y_{2}(0)$ plane in Fig. 12(b), which means a negative tool deflection $\left[y_{1}(0)<0\right]$ benefits the cutting stability. This basin is then pushed rightwards when $N \phi=\pi / 4$ so that a positive deflection $\left[y_{1}(0)>0\right]$ is preferred. Next, $N \phi=\pi / 2$ further enlarges the safe basin and moves it downwards, which decreases the corresponding SL as $\left(y_{10}, 0\right)$ is too close to the upper boundary of the white region. For a further increase of $N \phi$, the basin begins to shrink so that all of the GIM, IF, and SL are decreased to stay at very low levels, where the SL is even zero as $\left(y_{10}, 0\right)$ is not included in the safe basins. This unwanted safe level is kept until $N \phi=2 \pi$, which is the end of the spatial period of the wave.

To sum up, from the viewpoint of either the surface waviness of the workpiece or the tool bending deflection, the best tool engagement geometry is for $N \phi=\pi / 2$ and the worst is for $N \phi=3 \pi / 2$.

\section{CHATTER ELIMINATION BY IMPACT}

Up to now, the initial conditions for $t \in[-\tau, 0)$, which are the starting point and state at time zero have been regarded 
(a)


FIG. 13. (a) Schematic illustration of hammer striking of the cutting tool. With different points of striking $\psi$ and hitting strength $\Delta y_{2}$, the cutting chatter for $\Omega=0.24$ and $\eta_{1} / \eta_{\min }=4.55$ might (a, b) decay to zero or (c, d) return to the unstable cutting, respectively.

as major factors affecting the long-term cutting dynamics. These three variables would have played significant roles in any dynamical systems described by DDEs. By combining all of them, one can design a strategy to drive a system from one attractor to another. If the time-delayed initial condition is located in the basin of attraction of the stable cutting, the chatter is deemed as being quenched. As illustrated in Fig. 13(a), when the chatter in the UZs is present, an external impact introduced to the tool can cause a deviation from its chatter trajectory [37]. To eliminate the vibration, one should consider two questions: (i) When should the tool be struck? and (ii) How strong should the impact be?

The two questions are related to two variables, $\psi$ and $\Delta y_{2}$, depicted in Figs. 13(b) and 13(d), where an appropriate $\psi$ is selected to introduce an impact. On chatter trajectories, $\psi=0$ denotes the time instant when we obtain $y_{2}=0$ and $\dot{y}_{2}>0$, while the next time instant for $y_{2}=0$ and $\dot{y}_{2}>0$ is marked as $\psi=2 \pi$. For a periodic chatter, $\psi \in[0,2 \pi]$ is very representative, since it covers all time instants for introducing an impact. However, it should be noted here that the chatter is not completely periodic and $\psi \in[0,2 \pi]$ only partly answers the first question for the case of quasiperiodic chatter. Nonetheless, the result would be still very instructive. At the instant of impacting $(t=0)$, the tool velocity suddenly changes from $y_{2}(0)$ to $y_{2}(0)+\Delta y_{2}$, where $\Delta y_{2}$ depends on the impact strength. Correspondingly, the next basin of attraction is defined on the $\psi-\Delta y_{2}$ plane to reveal the effect of impacting.

Let us begin with a representative example calculated for $\Omega=0.24$ and $\eta_{1} / \eta_{\min }=4.55$. The impact $\Delta y_{2}=-1$ is introduced at $\psi=0.74$ as shown in Fig. 13(b), which suddenly changes the tool velocity $y_{2}(0)$ from 0.29 to -0.71 . Then
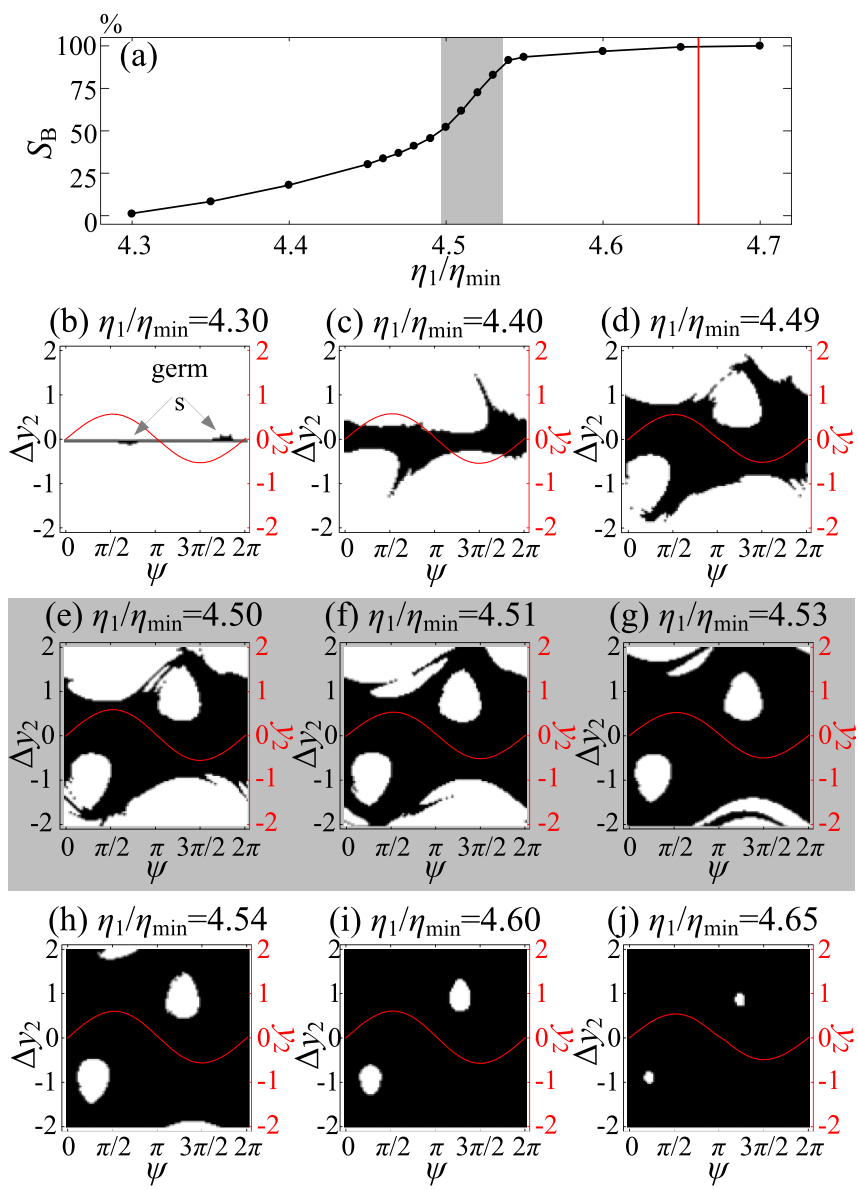

FIG. 14. (a) Evolution of $S_{B}$ with respect to the increase of $\eta_{1} / \eta_{\min }$ with typical basins of attraction for $\eta_{1} / \eta_{\min }=$ $4.30,4.40,4.49,4.50,4.51,4.53,4.54,4.60$, and 4.65 plotted in panels $(\mathrm{b}-\mathrm{j})$. The gray background for $\eta_{1} / \eta_{\min } \in(4.49,4.54)$ [corresponding with panels $(\mathrm{e}-\mathrm{g})]$ is added to marked the fastest evolution of $S_{B}$, which connect a moderate increase of $S_{B}$ on its left side and a slow grow of $S_{B}$ on its right side.

the chatter gradually decays to zero thereafter as shown in Fig. 13(c). By contrast, if the impact is $\Delta y_{2}=1$, the tool velocity $y_{2}(0)$ jumps to 1.29 as shown in Fig. 13(d) and then the response depicted in Fig. 13(e) returns to the chatter trajectory after the transient phase.

Next, in the span of $\psi \in[0,2 \pi]$, an impact with the strength of $\Delta y_{2} \in[-2,2]$ is introduced. As seen in Figs. 14(b)-14(j), if the chatter decays to zero, the corresponding point $\left(\psi, \Delta y_{2}\right)$ is marked in white, and the point is black if it returns to the chatter trajectory. Then, $S_{B}$ can be calculated from Eq. (9). Moreover, to clearly illustrate the time instant of striking, the time histories of $y_{2}$ are added in Figs. 14(b)-14(j), so that one can understand how the tool movement influences the effect of impact.

When $\Omega=0.24$, the influence of $\eta_{1} / \eta_{\min }$ on $S_{B}$ is shown in Fig. 14(a), where $S_{B}$ increases monotonously toward $100 \%$ with respect to the increase of $\eta_{1} / \eta_{\min }$. For $\eta_{1} / \eta_{\min } \in$ $[4.30,4.54], S_{B}$ grows in a moderately rate until it enters the grey zone $\left[\eta_{1} / \eta_{\min } \in(4.49,4.54)\right]$ to experience its fastest growth before it levels off thereafter. 
When $\eta_{1} / \eta_{\min }=4.30, S_{B}$ is almost zero, and its corresponding basins of attraction in Fig. 14(b) has only a small black region along the axis $\Delta y_{2}=0$, which means no impact. However, this region has two small "germs," which slightly increase the potentials for chatter occurrence. The locations of the "germs" remind us that when the cutting tool starts to decelerate, a slight impact in the same direction would not destroy the chatter.

As $\eta_{1} / \eta_{\text {min }}$ increases toward the boundary, the black region simultaneously expands upwards and downwards, as shown in Figs. 14(b)-14(d). Moreover, the left edges of the two "germs" extend much faster than any other zones, which incline leftwards and then fold back to wrap two "islands." Due to the nonlinearity in the regenerative cutting force, the left boundaries of the "islands" in Fig. 14(d) becomes fractal so that the white regions interweaves with the black one. As soon as the two "islands" have been isolated from other white regions, the increase of $S_{B}$ enters the gray region for its fastest growth. Corresponding basins of attraction are displayed in Figs. 14(e)-14(g), where the white regions, except the two "islands," shrink fast and move away from the axis $\Delta y_{2}=0$. Thereafter, the change of the "islands" is so slow that the increase of $S_{B}$ is almost invisible when it leaves the gray zone.

Evolution of $S_{B}$ with respect to $\eta_{1} / \eta_{\text {min }}$ depicted in Fig. 14 shows the robustness of the "islands" for the two following reasons. On the one hand, for $\eta_{1} / \eta_{\min } \in[4.55,4.65]$, the change of $S_{B}$ is extremely slow since the "islands" are the only white regions on the plane. On the other hand, as seen in Figs. 14(b)-14(d), when the "islands" connect with other white regions, the change of $S_{B}$ for $\eta_{1} / \eta_{\min } \in[4.30,4.49]$ has only a moderate rate, which is much slower than that in the gray region where the "islands" are isolated. That is to say, the "islands" slow down the evolution rate of the other white regions. The robustness of the "islands" reminds us that one can utilize the "islands" to design a controller quenching the cutting chatter by even a single strike. Meanwhile, the locations of the "islands" indicate that the impact should has the strength around $\left|\Delta y_{2}\right| \approx 1$ and is opposite to the tool motion during its acceleration. Such a controller would be efficient even when the parameter values in the UZs are very close to the linearly stable boundary.

\section{CONCLUSIONS}

We have proposed a systematic method to predict the longterm dynamics of time-delayed systems for bistable regions considering the delayed states, starting point, and state at time zero as three major factors. More specifically for the cutting process, the initial workpiece surface profile, cutting geometry, and tool bending deflection determine the long-term cutting dynamics in UZs.
The time-delayed initial condition was regarded as the first factor affecting the long-term dynamics. For the cutting process, the state for $t \in[-\tau, 0)$ is determined by the waviness of the workpiece surface. It was revealed that the probability of chatter occurrence increases not only near the primary harmonics but also near its sub- and superharmonics. This unexpected phenomenon provides additional means to place the system dynamics governed by DDEs on a desired oscillating orbit.

When the parameter values of the cutting process move toward the stability boundaries in the UZs, the basin stability of the chatter increases slowly at first but very fast at last, yielding a "boundary layer" in the immediate vicinity of the stability boundaries. The "boundary layer" is very thin that most area of the UZ (over $90 \%$ ) has no more than $10 \%$ probability of chatter occurrence.

Next a consideration was given to the cutting geometry, which determines the second important factor influencing the long-term dynamics, the starting point. It was found that the basin stability of the chatter is much smaller when the cutting geometry is on the top of wave crests $(N \phi=\pi / 2)$, while the chatter is prone to occur when the cutting starts at $N \phi=3 \pi / 2$.

The last consideration was given to the tool deflection at the instant of tool-workpiece engagement, which is exactly the same as the conventional basins of attraction used in ODEs. Accordingly, the cutting safety has been estimated by three different measures, GIM, IF, and SL. GIM and IF evaluate the overall safety of the cutting, while SL assesses the safety around $\left[y_{1}(0), y_{2}(0)\right]=\left(y_{10}, 0\right)$. Influence of $N \phi$ on GIM, IF, and SL showed a consistency with previous discussion on the cutting geometry. Moreover, it was revealed that SL could be zero when $N \phi \approx 3 \pi / 2$, indicating unsafe cutting.

Basins of attraction cannot only be used to track the longterm dynamics but also can be used to drive it to a desired attractor. To quench the chatter by introducing an impact at the right time and with the right strength, the basin stability on the $\psi-\Delta y_{2}$ plane was studied, where the "islands" give an opportunity to cease the chatter by even a single strike when the cutting has been selected close to the linearly stability boundary. Such an impact is suggested to be of the strength $\left|\Delta y_{2}\right| \approx 1$ and opposite to the moving direction of the cutting tool when it accelerates its speed from zero to its maximum.

\section{ACKNOWLEDGMENTS}

This research is supported by National Natural Science Foundation of China under Grants No. 11502048, No. 11772229, and No. 11572224, and Fundamental Research Funds for the Central Universities under Grant No. ZYGX2015KYQD033.
[1] A. Roxin and A. Compte, Phys. Rev. E 94, 012410 (2016).

[2] V. Odent, E. Louvergneaux, M. G. Clerc, and I. Andrade-Silva, Phys. Rev. E 94, 052220 (2016).

[3] V. V. Semenov, A. B. Neiman, T. E. Vadivasova, and V. S. Anishchenko, Phys. Rev. E 93, 052210 (2016).
[4] T. Gross, Carlos J. Dommar D’Lima, and B. Blasius, Phys. Rev. Lett. 96, 208701 (2006).

[5] A. Reid, F. Lechenault, S. Rica, and M. Adda-Bedia, Phys. Rev. E 95, 013002 (2017).

[6] A. N. Pisarchik and U. Feudel, Phys. Rep. 540, 167 (2014). 
[7] A. N. Pisarchik and B. K. Goswami, Phys. Rev. Lett. 84, 1423 (2000).

[8] Z. Chen and X. Liu, Phys. Rev. E 95, 012208 (2017).

[9] H. Kim, S. H. Lee, and P. Holme, Phys. Rev. E 93, 062318 (2016).

[10] J. K. Hale, Functional Differential Equations (Springer, Berlin, 1971).

[11] H. Wang, H. Hu, and Z. Wang, Int. J. Bifurcat. Chaos 14, 2753 (2004).

[12] K. Höhne, H. Shirahama, C.-U. Choe, H. Benner, K. Pyragas, and W. Just, Phys. Rev. Lett. 98, 214102 (2007).

[13] H. Shang and J. Xu, Chaos Solitons Fract. 41, 1880 (2009).

[14] J. C. Ji, J. Sound Vibration 349, 299 (2015).

[15] S. Leng, W. Lin, and J. Kurths, Sci. Rep. 6, 1 (2016).

[16] S. T. S. Bukkapatnam, A. Lakhtakia, and S. R. T. Kumara, Phys. Rev. E 52, 2375 (1995).

[17] G. Orosz, R. E. Wilson, and B. Krauskopf, Phys. Rev. E 70, 026207 (2004).

[18] C.-Y. Kao, C.-W. Shih, and C.-H. Wu, Physica D (Amsterdam) 328, 21 (2016).

[19] S. Zhang, J. Xu, and K. W. Chung, Neurocomputing, 266, 42 (2017).

[20] E. V. Grigorieva and S. A. Kaschenko, Physica D (Amsterdam) 291, 1 (2015).

[21] B. Thakur, D. Sharma, A. Sen, and G. L. Johnston, Phys. Rev. E 95, 012204 (2017).

[22] Z. Levnajić, Phys. Rev. E 84, 016231 (2011).
[23] Z. Dombovari and G. Stépán, Philos. Trans. R. Soc. London A 373, 20140409 (2015).

[24] A. Depouhon, V. Denoël, and E. Detournay, Physica D (Amsterdam) 258, 1 (2013).

[25] Y. Yan, J. Xu, and M. Wiercigroch, Nonlinear Dynam. 86, 283 (2016).

[26] T. G. Molnár, T. Insperger, S. John Hogan, and G. Stépán, J. Comput. Nonlin. Dyn. 11, 051008 (2016).

[27] Z. Dombovari, R. E. Wilson, and G. Stépán, Proc. R. Soc. London A 464, 3255 (2008).

[28] J. Xu and K. W. Chung, Physica D (Amsterdam) 180, 17 (2003).

[29] M. Wiercigroch and E. Budak, Philos. Trans. R. Soc. London A 359, 663 (2001).

[30] H. M. Shi and S. A. Tobias, Int. J. Mach. Tool D. R. 24, 45 (1984).

[31] G. Stépán, Philos. Trans. R. Soc. London A 359, 739 (2001).

[32] Y. A. Kuznetsov, Elements of Applied Bifurcation Theory (Springer-Verlag, New York, 2000).

[33] U. Chaudhuri and A. Prasad, Phys. Lett. A 378, 713 (2014).

[34] G. Rega and S. Lenci, J. Vibrat. Control 14, 159 (2008).

[35] S. Lenci and G. Rega, Physica D (Amsterdam) 240, 814 (2011).

[36] S. Lenci, D. Orlando, G. Rega, and P. B. Gonalves, Chaos 22, 047502 (2012).

[37] C. von Loewenich, H. Benner, and W. Just, Phys. Rev. Lett. 93, 174101 (2004). 